\title{
Advancing understanding and prediction in multiple stressor research through a mechanistic basis for null models
}

\author{
Ralf B. Schäfer ${ }^{1}$ (D) | Jeremy J. Piggott ${ }^{2}$
}

${ }^{1}$ Quantitative Landscape Ecology, Institute for Environmental Sciences, University Koblenz-Landau, Landau in der Pfalz, Germany

${ }^{2}$ School of Natural Sciences, Trinity College Dublin, The University of Dublin, Dublin, Ireland

\section{Correspondence}

Ralf B. Schäfer, Quantitative Landscape Ecology, Institute for Environmental Sciences, University Koblenz-Landau, Landau in der Pfalz, Germany.

Email: schaefer-ralf@uni-landau.de

Funding information

Deutsche Forschungsgemeinschaft, Grant/ Award Number: SCHA 1720/17-1

\begin{abstract}
Global environmental change is driven by multiple anthropogenic stressors. Conservation and restoration require understanding the individual and joint action of these stressors to evaluate and prioritize management measures. To date, most studies on multiple stressor effects have sought to identify potential stressor interactions, defined as deviations from null models, and related meta-analyses have focused on quantifying the relative proportion of stressor interactions across studies. These studies have provided valuable insights about the complexity of multiple stressor effects, but remain largely devoid of a theoretical framework for null model selection and prediction of effects. We suggest that multiple stressor research would benefit by (1) integrating and developing additional null models and (2) selecting null models based on their mechanistic assumptions of the stressor mode of action and organism sensitivities as well as stressor-effect relationships for individuals and populations. We present a range of null models and outline their underlying assumptions and application in multiple stressor research. Moving beyond mere description requires multiple stressor research to shift its focus from identifying statistically significant interactions to the use and development of mechanistic (null) models. Justified selection of the appropriate null model is a first step to achieve this.
\end{abstract}

\section{KEYWORDS}

antagonism, mechanism, mixtures, multiple stress, null models, stressors, synergism

Glossary: antagonism, interaction between stressors that results in a lesser combined effect than that predicted by a null model; ecological entity, individual, population, community or ecosystem; effect limit, maximum effect size for a response (e.g. 100\% mortality, zero growth or reproduction); effect type, a measurable property of an ecological entity that is affected by a stressor such as growth, reproduction or survival of individuals termed endpoint in ecotoxicology.; effect, a change in a response resulting from a cause, typically from a stressor in the context of multiple stressor research; interaction, modification of (1) a stressor's intensity or (2) the sensitivity of an organism towards this stressor by another stressor or multiple other stressors (see introduction for example); null model for multiple stressors, a model that predicts the joint effect assuming the absence of interactions; response, a quantifiable biological or ecological property (e.g. survival, abundance, respiration), constituting the response variable during data analysis. We use response in this narrow sense for terminological clarity, that is, we exclusively use "effect" to refer to a stressor-driven change in a property in comparison to a reference state (e.g. control in experiment); sensitivity distribution, absolute frequency or relative frequency (i.e. density) distribution of sensitivities within populations or communities. Not to be confused with the concept of species sensitivity distribution (SSD) used in ecological risk assessment, which represents a cumulative distribution. Note that in our context, for mortality as effect, the stressor-effect relationship represents the cumulative distribution of the sensitivity density distribution.; sensitivity, the minimum stressor intensity at which an ecological entity shows an effect; stress capacity, the opposite of sensitivity in a conceptual sense, that is, the maximum stressor intensity that an ecological entity can tolerate without showing an effect (introduced in Liess, Foit, Knillmann, Schäfer, \& Liess, 2016); stressor intensity, quantifiable strength of a stressor (e.g. wind speed, concentration, temperature); stressor mode of action (SMOA), describes how a stressor affects an organism and informs whether stressors act similarly or dissimilarly. We tentatively distinguish, from an idealized perspective, three modes of action: stressors acting (1) from the outside on the whole organism such as predation, flood or storm, leading to physical destruction or displacement, (2) from the inside or outside relatively nonspecifically on the physiology of an organism such as competition, salinity and heat (but also predation, floods or storms), leading to physiological effects through energy costs or (3) from the inside relatively specifically on the physiology of an organism such as many toxicants, leading to physiological effects through alteration of specific physiological processes; Stressor, a natural or anthropogenic environmental factor that can affect an individual; synergism, interaction between stressors that results in a stronger combined effect of stressors than that predicted by a null model. 


\section{1 | INTRODUCTION}

The current epoch is characterized by the unprecedented impact of human activities on ecosystems through multiple anthropogenic stressors (Lewis \& Maslin, 2015). The co-occurrence of these stressors is rather the norm (Schäfer, Kühn, Malaj, König, \& Gergs, 2016) and many studies in a variety of ecosystems have examined interaction effects, for example, antagonistic and synergistic effects (Côté, Darling, \& Brown, 2016). Studies to date have largely focused on twostressor interactions (Griffen, Belgrad, Cannizzo, Knotts, \& Hancock, 2016; but see for example Piggott, Salis, Lear, Townsend, \& Matthaei, 2015), whereby one stressor modifies (1) the second stressor's intensity (e.g. change in toxicant concentration by UV radiation) or (2) the sensitivity of organisms towards the other stressor (e.g. change in susceptibility to predation (stressor 1 ) through behavioural effects of stressor 2 such as reduced mobility; for details see Kroeker, Kordas, \& Harley, 2017). Stressor interactions are determined in reference to a null model that predicts the joint effect assuming the absence of interactions, that is, the stressors are operating independently. If this is the case, the joint effect can be predicted from knowledge of single stressor effects (Kendler \& Gardner, 2010; Piggott, Townsend, \& Matthaei, 2015). If the joint effect deviates (more than an acceptable margin, further details below) from the null model as a result of antagonistic or synergistic interaction between stressors, more complex models accounting for stressor interactions may be required for reliable prediction (Thompson, MacLennan, \& Vinebrooke, 2018).

Recent meta-analyses of studies on two-stressor interactions across ecosystems (and their constituent ecological entities) displayed a high variability in the prevalence of antagonistic or synergistic interactions (Côté et al., 2016). For example, a meta-analysis of 148 studies on the interactions of chemicals with natural stressors, predominantly focusing on single species experiments on the physiological and population level, reported $68 \%$ of studies with synergism (Holmstrup et al., 2010). By contrast, interactions of multiple invaders on populations and communities were rarely synergistic $(4 \%$, $n=45$ ) (Jackson, 2015), and of 286 responses of freshwater ecosystems for different paired stressors, the interactions were more frequently antagonistic (41\%) than synergistic (28\%) (Jackson, Loewen, Vinebrooke, \& Chimimba, 2016). Moreover, a meta-analysis of 171 studies on multiple stressor interactions in the marine system found that two-stressor interactions were often context dependent (67\%), that is, conditioned by a third variable, which, compared to the ambient level, mostly (75\%) exacerbated the negative effect (Crain, Kroeker, \& Halpern, 2008). However, the variability in the interaction types was not well explained by covariables such as ecosystem type, stressor type, taxonomic group and type of study (Côté et al., 2016), although antagonism rarely occurred on the physiological level and synergism was uncommon on the community level, respectively. This in turn limits our ability to predict the effects of multiple stressors as well as to effectively manage ecosystems (Brudvig, 2017).

Although the complexity of the systems in which responses are measured may hamper generalizable explanations and prediction, differences in (1) interaction classification frameworks, which define mathematically what constitutes a synergistic and antagonistic interaction (for details see Piggott, Townsend et al., 2015; Hale, Piggott, \& Swearer, 2017) and (2) the type of null models underlying such frameworks add to the variability in reported interaction types, thus limiting comparability. The selected null model should ideally be underpinned by a mechanistic understanding of single/multiple stressor effects, determined by the stressor/s mode of action and stressor-effect relationship/s (Figure 1a), although to date this has been largely overlooked due to statistical convenience. In this article, we address this issue by providing an overview of a range of null models, including those rarely considered by ecologists and discuss their underlying mechanistic assumptions and study design requirements for their application. In addition, we provide null model selection guidelines. We focus our examples on interactions between two stressors and suggest that before higher order interactions are tackled, we should strive to understand two-way interactions in terms of (1) the guidelines for null model selection and (2) novel models that can predict the effects of potentially interacting stressors, which are not covered by any of the current frameworks.

\section{2 | MECHANISTICALLY BASED NULL MODELS FOR MULTIPLE STRESSORS}

Stressors generally act on individuals (Maltby, 1999). The stressor can have physical (e.g. flood dislocating an organism, storm removing leafs from a tree) and physiological (e.g. toxicants acting on the physiology of an organism) effects, depending on the stressor mode of action. The magnitude of the effect depends on the stressor intensity and the sensitivity of an individual (Liess et al., 2016)(Figure 1a). For multiple individuals, the average magnitude of effect, which is often reported (e.g. average growth reduction, average mortality), depends, beside the stressor intensity, on the sensitivity distribution across individuals. Although most studies report only summary statistics (e.g. confidence interval for average growth reduction) and few studies provide the raw data to derive this distribution, it can, at least if the effect is mortality, be derived from the stressor-effect relationship (Figure 1a). Although the term stressorresponse relationship has been used more frequently, we suggest that establishing the relationship with the effect is more consistent with null model notations relating to effects (see Côté et al., 2016). Idealized shapes of two commonly employed stressor-effect relationships (linear and sigmoidal) for populations are presented in Figure 1a. For mortality as the effect, a linear stressor-effect relationship implies that the sensitivity distribution is uniform. This is, because for a linear relationship, each unit increase in stressor intensity removes an equal-sized fraction of the population (e.g. a two-fold increase in stressor intensity yields to a two-fold increase in the affected fraction), which translates to a uniform density distribution of individuals' sensitivities. Conversely, a sigmoid stressor-effect relationship implies that the sensitivities in a population follow a unimodal density distribution (Figure 1a). 
(a)

\section{Single stressor}

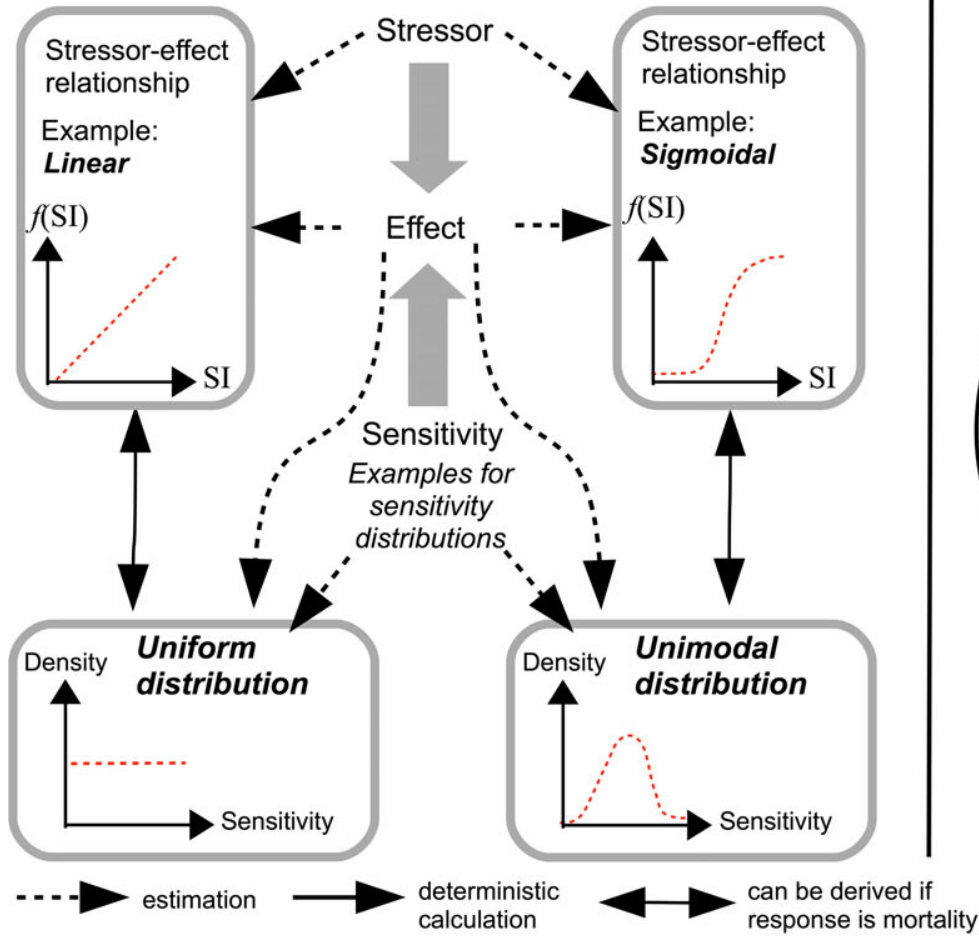

(b)

\section{Multiple stressor null models}

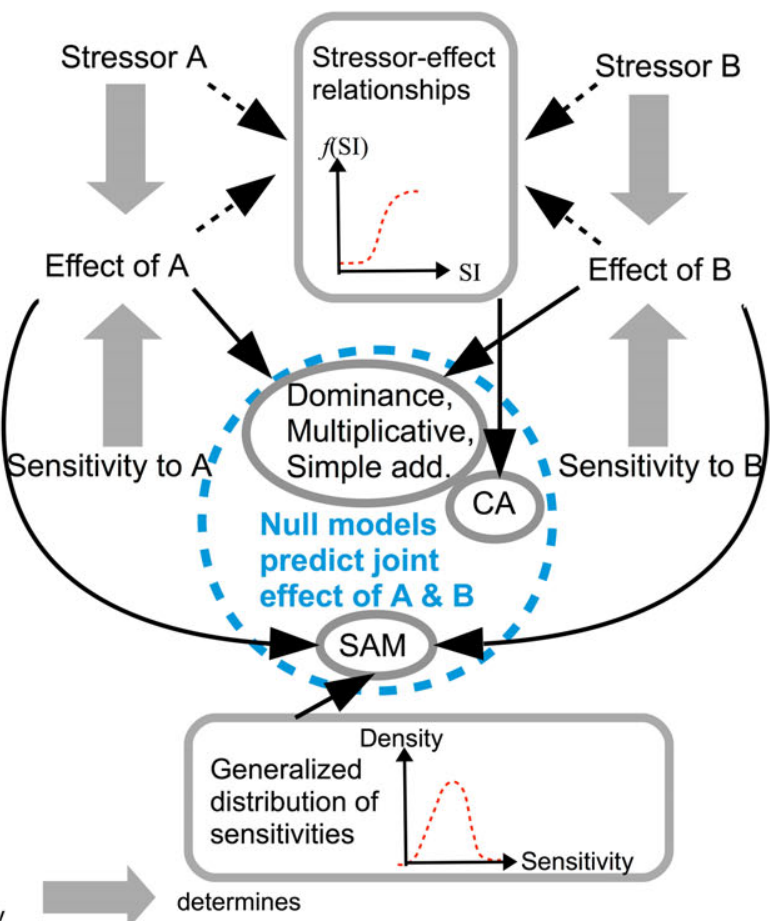

FIGURE 1 Conceptual representation of (a) elements involved in the determination of single stressor effects and in the estimation of stressor-effect relationships as well as sensitivity density distributions and (b) their relationship with null models for multiple stressor effects. Thin and thick arrows indicate mathematical and causal relations respectively. To enhance visual clarity, only most relevant relations are displayed. "Estimation" (dashed arrow) refers to parameter estimation from observations through fitting of a statistical model. "Deterministic calculation" (solid arrow) refers to calculations based on deterministic equations as in Table 1. Null models calculated from same input data are placed into the same circle to enhance visual clarity. SI: Stressor intensity; $f(S I)$, Stressor effect as a function of SI; Simple add., Simple addition; CA, Concentration addition; SAM, Stressor addition model

In the context of two stressors, the stressor-effect relationship is described by a surface (see for example Lange, Townsend, \& Matthaei, 2014) and its shape will depend on potential interactions between stressors. As mentioned earlier, null models assume that such interactions are absent and predict the joint effect of two stressors based on the effects of single stressors (Figure $1 \mathrm{~b}$, details on null models given below).

Many ecological studies have employed the simple addition model (Table 1) (Crain et al., 2008; Jackson et al., 2016), presumably because this represents the most parsimonious null model. Moreover, the simple addition model is imposed when using analysis of variance (ANOVA), which is often selected for statistical analysis of response data from factorial studies (Dunne, 2010; Quinn \& Keough, 2002). This imposition of null models associated with selecting a statistical model is frequently overlooked by researchers (Griffen et al., 2016), who typically select the statistical model based on the type of response variable rather than the knowledge of the potential mechanisms underlying joint stressor effects. For example, ANOVA imposes the simple addition model, whereas the same statistical model for log-transformed data imposes the multiplicative null model (see below for details). A recent analysis suggested that this change in the null model is ignored in approximately $1 / 3$ of marine multiple stressor studies (Griffen et al., 2016). However, the simple addition and multiplicative null model can result in different interaction classifications for many data sets and have different underlying mechanistic assumptions, although both models share the similarity that the stressors can be conceptualized as acting sequentially (cf. Vinebrooke et al., 2004). The simple addition model assumes a linear stressor-effect relationship (cf. Figure 1a). If mortality is the effect and the stressors are conceptualized as acting sequentially, the simple addition model translates into the assumption that the sensitivities of single individuals in the population are strongly negatively correlated, for example, individuals sensitive to stressor A would be tolerant to stressor B and vice versa. Then, each stressor would independently kill a fraction of the population (Figure 2, Table 1). By contrast, for the same conditions, the multiplicative null model implies the assumption that the sensitivities are noncorrelated for mortality as the effect (Figure 2). For nonlethal responses (e.g. growth or reproduction), the null models do not imply the specific assumptions regarding the correlation of sensitivities outlined above for mortality. In other words, the respective null models match the data if the sensitivities are correlated as is described for mortality 
TABLE 1 Overview on different null models for two stressors or chemicals formulated according to the notation suggested by Howard and Webster (2009) with required input data for calculation

\begin{tabular}{|c|c|c|c|}
\hline $\begin{array}{l}\text { Ecological } \\
\text { model }\end{array}$ & $\begin{array}{l}\text { (Eco)toxicological } \\
\text { model }\end{array}$ & $\begin{array}{l}\text { Null model equation for multiple } \\
\text { stressor research }\end{array}$ & Required input data \\
\hline Simple addition & - & $f_{A B}\left(S I_{A}, S I_{B}\right)=f_{A}\left(S I_{A}\right)+f_{B}\left(S I_{B}\right)$ & Effects for $A$ and $B$ \\
\hline Dominance & - & $f_{A B}\left(S I_{A}, S I_{B}\right)=\max \left(f_{A}\left(S I_{A}\right), f_{B}\left(S I_{B}\right)\right)$ & Effects for $A$ and $B$ \\
\hline- & $\begin{array}{l}\text { Concentration } \\
\text { addition }^{\text {a }} \text { (CA) }\end{array}$ & $f_{A B}\left(S I_{A}, S I_{B}\right)=f_{A}\left(S I_{A}+\gamma S I_{B}\right)$ with $\gamma=S I x_{A} / S x_{B}$ & $\begin{array}{l}\text { Stressor-effect relationship for } A \text {, stressor intensity for } A \text { and } B \\
\text { and stressor intensities } S \mid x_{A} \text { and } S \mid x_{B} \text { that result in the same } \\
\text { effect size } x\left(x=f_{A}\left(S \mid x_{A}\right)=f_{B}\left(S \mid x_{B}\right)\right) \text {, where } x \text { is typically } \\
\text { selected as half the effect limit }\end{array}$ \\
\hline- & $\begin{array}{l}\text { Stressor addition } \\
\text { (SAM) }\end{array}$ & $\begin{array}{l}f_{A B}\left(S I_{A}, S I_{B}\right)=F_{\text {Strcap }}\left(F_{\text {Strcap }}{ }^{-1}\left(f_{A}\left(S I_{A}\right)\right)\right. \\
\left.\quad+F_{\text {Strcap }}{ }^{-1}\left(f_{B}\left(S I_{B}\right)\right)\right)\end{array}$ & $\begin{array}{l}\text { Generalized density function for the distribution of the stress } \\
\text { capacity } f_{\text {Strcap }}{ }^{c} \text { and effects for } A \text { and } B\left(f_{A}\left(S I_{A}\right) \text { and }\left(f_{B}\left(S I_{B}\right)\right)\right.\end{array}$ \\
\hline
\end{tabular}

${ }^{a}$ Note that under generalized concentration addition the assumption that $\gamma$ is constant $\left(=\mathrm{S}_{\mathrm{A}} \mathrm{A} / \mathrm{S} \mid \mathrm{x}_{\mathrm{B}}\right)$ is dropped (for details see Howard \& Webster, 2009).

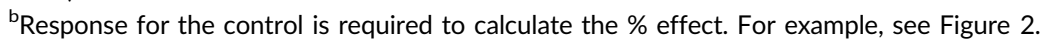

${ }^{c}$ visualized in Figure $1 \mathrm{~b}$ as sensitivity distribution for the purpose of simplicity and consistency within Figure 1.

$\mathrm{SI}_{\mathrm{A}}$, Stressor Intensity of stressor $\mathrm{A} ; \mathrm{SI}_{B}$, Stressor Intensity of stressor $\mathrm{B} ; \mathrm{f}_{\mathrm{A}}\left(\mathrm{SI} \mathrm{I}_{\mathrm{A}}\right)=$ Effect of stressor $\mathrm{A}$ for intensity $\mathrm{SI}_{\mathrm{A}}$ and a given stressor-effect relationship $f_{A} ; f_{B}\left(S I_{B}\right)=$ Effect of stressor $B$ for intensity $S I_{B}$ and a given stressor-effect relationship $f_{B} ; f_{A B}\left(S I_{A}\right.$, $\left.S I_{B}\right)=$ Joint effect of stressors $A$ and $B$ for intensities $\mathrm{SI}_{A}$ and $S \mathrm{~S}_{B}$ given a stressor-effect relationship $f_{A B} ; f_{\text {strcap }}=$ generalized density function for the density distribution of the stress capacity (Stresscap), $F_{\text {Strcap }}$ represents the cumulative density function.

(Figure 2), but they can also match data with different underlying sensitivity correlations.

Another model type frequently used in ecological studies is dominance (also termed comparative effects model) that predicts that the effect of stressors in combination is equal to that of the worst or dominant stressor (Table 1; Folt, Chen, Moore, \& Burnaford, 1999). Under such model, the dominant stressor takes precedence over lesser stressors that have no additional impact. Mechanistically, if the stressors are conceptualized as acting sequentially, this null model implies the assumption that stressor sensitivities are strongly positively correlated (Figure 2).

Similar and additional null models have been developed in scientific disciplines such as toxicology and ecotoxicology dealing with the effects of chemicals on biological systems. We believe that the models and mechanisms developed in ecotoxicological research represent approaches that may, at least partially, be adopted by ecologists for predicting effects of multiple stressors. The two fundamental null models to study the effects of multiple chemicals that have been developed in the first half of the 20th century are effect addition (EA), which corresponds to the above-mentioned multiplicative null model used in ecological studies, and concentration addition (CA) (Backhaus \& Faust, 2012). Note that interchangeable terms have been used for these models in toxicology and ecotoxicology (see Cedergreen, 2014). The EA model (Bliss, 1939) assumes additivity of two effects in terms of the probabilistic sum, which accounts for the probability that, in the case of mortality, an individual is killed by both chemical concentrations by subtracting the product of their effects:

$$
f_{A B}\left(c_{A}, c_{B}\right)=f_{A}\left(c_{A}\right)+f_{B}\left(c_{B}\right)-f_{A}\left(c_{A}\right) f_{B}\left(c_{B}\right)
$$

where $f_{i}\left(c_{i}\right)$ is the effect of concentration $c_{i}$ of a chemical $i$ for a given concentration-effect relationship $f_{i}$. The EA model is typically used if chemicals are expected to act on different target sites in the organism (Escher, 2013). For example, the chemical A inhibits a neurotransmitter, whereas the chemical $B$ inhibits the mitochondrial energy transport. Substituting the concentrations $c$ with stressor intensities (SI) in Equation (1) proves the general equivalence of the multiplicative and the EA model. Therefore, the assumption for the multiplicative model, that the sensitivities are noncorrelated (Figure 2), also applies to the EA model. The EA and multiplicative model are generally to be used with data that have been transformed to proportions (\%), where the control is typically set to $100 \%$ (e.g. growth, survival)(Folt et al., 1999).

The CA model (Loewe \& Muischnek, 1926) assumes that the concentrations $c$ of chemicals are exchangeable if scaled by their potency. In analogy to two stressors, we consider the case of two chemicals A and B (notation following Howard \& Webster, 2009), for which exchangeable potency can be expressed as:

$$
1=\frac{C_{A}}{E C x_{A}}+\frac{C_{B}}{E C x_{B}}
$$

where $\mathrm{ECx}_{\mathrm{A}}$ and $\mathrm{ECx}_{\mathrm{B}}$ are the concentrations, where $\mathrm{x} \%$ effect is observed under standardized conditions. Thus, $E C x_{A}$ can be expressed as:

$$
E C x_{A}=c_{A}+\frac{E C x_{A}}{E C x_{B}} c_{B}
$$

Equation 3 only describes the exchangeability between chemicals but lacks a link to effects of the concentrations, that is, $f_{i}\left(c_{i}\right)$. Under the assumption of exchangeability, the joint effect $f_{A B}\left(c_{A}, c_{B}\right)$ can be expressed in terms of $f_{A}\left(E C x_{A}\right)$ :

$$
f_{A B}\left(c_{A}, c_{B}\right)=f_{A}\left(E C x_{A}\right)=f_{A}\left(c_{A}+\frac{E C x_{A}}{E C x_{B}} c_{B}\right)
$$

The CA model is typically used if chemicals are expected to have the same mode of action (Escher, 2013). For example, both 


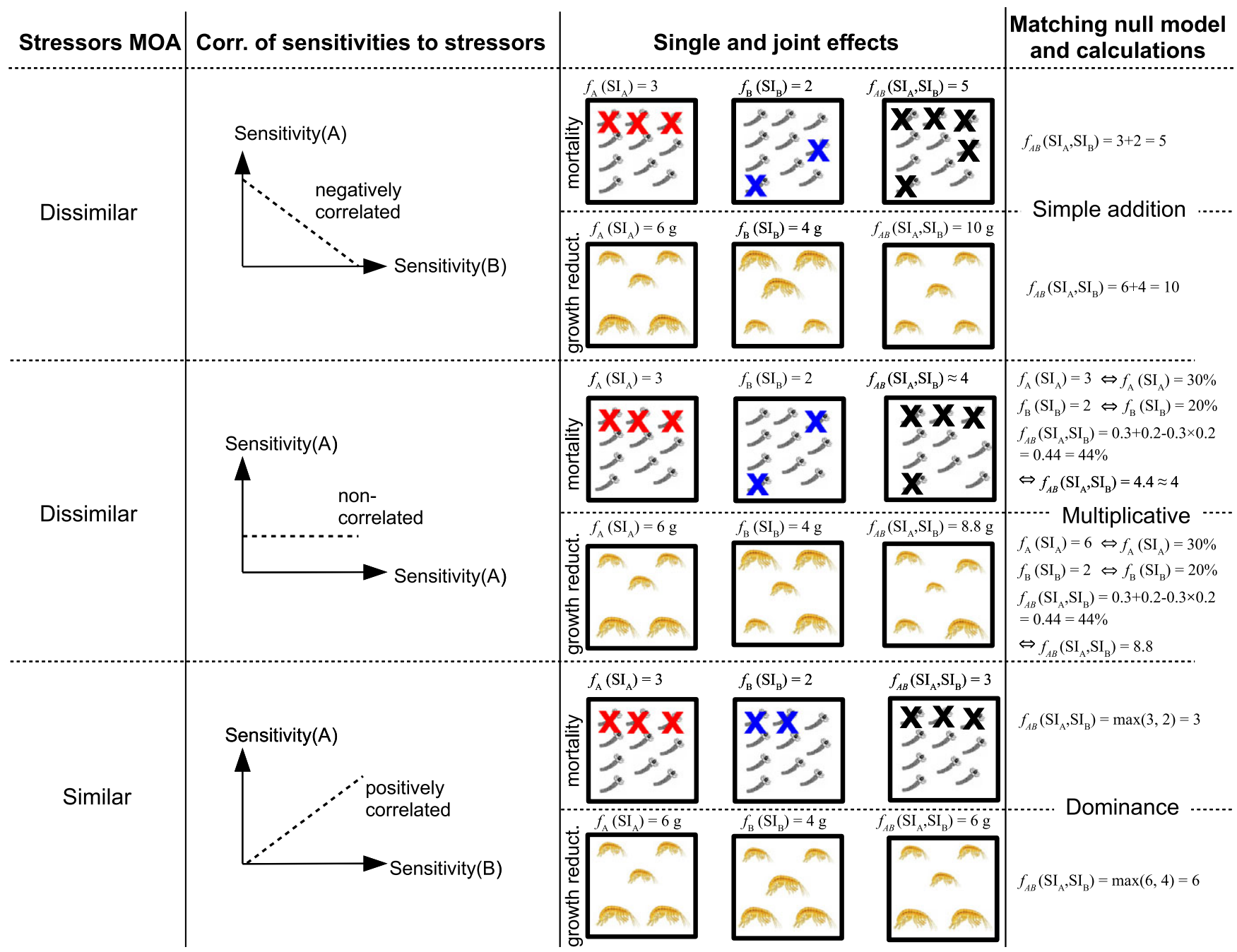

FIGURE 2 Implications of stressor mode of action (SMOA, see glossary) and correlations (corr.) of sensitivities for joint effects and matching null models. For details on stressor notation and formulas for calculation, see Table 1. The single and joint effects are exemplified for survival of caddisfly larvae and growth of gammarids, numbers above organisms refer to a reduction in survival (hereafter and in figure: mortality) in number of dead individuals and reduction in growth in g biomass respectively. The control (100\%) survival and growth are 10 individuals and $20 \mathrm{~g}$ respectively. Note that if the effect is expressed as (\%) of control in the multiplicative model, the formula notation $f_{A}\left(S_{A}\right) \times f_{B}\left(S_{B}\right)$ as in analysis of variance $(A \times B$, for details see Folt et al., 1999) applies (e.g. $70 \%$ and $80 \%$ survival of control:

$0.7 \times 0.8=0.56=56 \%$, which is equivalent to the result in Figure 2: $100 \%-56 \%=44 \%$ mortality). Mortality visualized as crosses, a reduction in growth as smaller body sizes. Individuals are identifiable through their position in the box. The stressors are conceptualized as acting sequentially and are interchangeable, that is, mathematically commutative. CA and SAM not included because their calculation requires additional input data besides the effects for A and B (Table 1), see Liess et al. (2016) for visualization

chemicals $A$ and $B$ in the mixture inhibit the same neurotransmitter. No directly corresponding model used in ecology has been developed, but the model can be adopted for nonchemical stressors, by translating the concentrations $c$ to stressor intensities (SI), and effect concentrations ECX to effect levels SIX $\mathrm{x}_{\mathrm{A}}$ and $\mathrm{SIx}_{\mathrm{B}}$ (see Table 1). The translated model inherits the assumption that the stressors have a similar or shared mode of action and, consequently, that the sensitivities to the different stressors are similar. An important difference to the null models discussed above is that the CA model relies on a stressor-effect relationship. It assumes that different stressor intensities can be converted into the intensities of one of the stressors, for example, stressor A. The joint effect is then given using the sum of stressor intensities as input for the stressor-effect function $f_{\mathrm{A}}$
(Table 1), which also implies that the stressors act simultaneously. The surface of the effects for two stressors is reduced to a curve or line. Note that the simple addition and dominance models can be regarded as special cases of CA. Simple addition is derived from CA if both stressors have the same potency $(\gamma=1)$ and shape of the stressor-effect relationship $\left(f_{\mathrm{A}}=f_{\mathrm{B}}\right)$ and if this stressor-effect relationship is linear $\left(f\left(\mathrm{SI}_{\mathrm{A}}+\mathrm{SI}_{\mathrm{B}}\right)=f\left(\mathrm{SI}_{\mathrm{A}}\right)+f\left(\mathrm{SI}_{\mathrm{B}}\right)\right)$. Similarly, the dominance model is derived by setting the potency of the stressor with the lesser effect to zero $(\gamma=0)$. However, from the differences in perspectives, with stressors conceptualized as acting sequentially for dominance and simple addition but as acting simultaneously for CA, follows that the assumptions of the null models relate to different phenomena and are not directly comparable. 


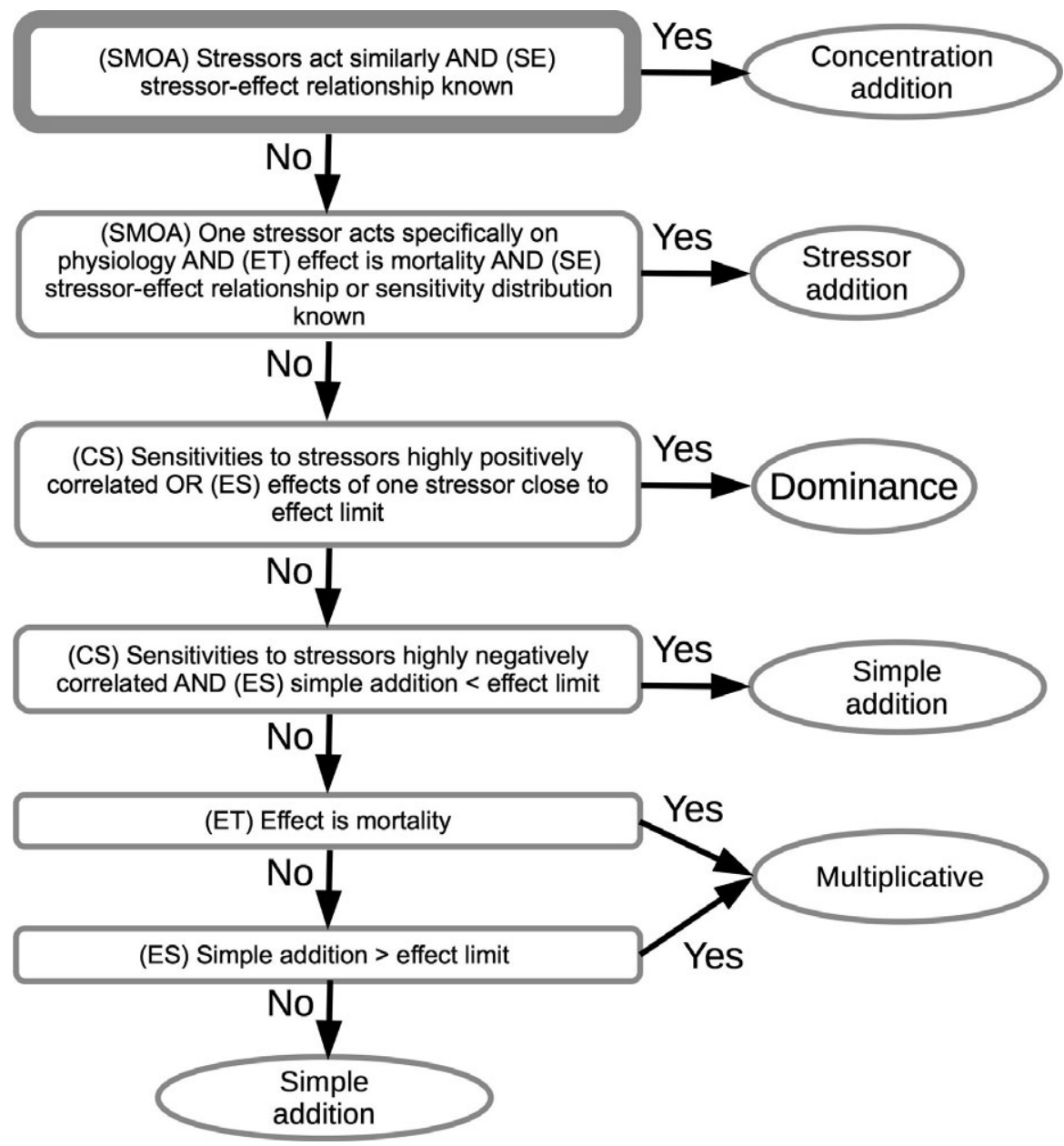

FIGURE 3 Guidelines for the selection of a null model for prediction of joint stressor effects on individuals (or populations, but see end of section 3 for caveats). Stressor notation follows Table 1. Stressor sensitivity correlations for simple addition, dominance and multiplicative null model conceptualized under the perspective of sequential stressor action (cf. Figure 2). For details on stressor modes of action, see glossary. Terms in brackets indicate the required information. SMOA, stressor mode of action (see glossary); CS, correlation of sensitivities; SE, stressoreffect relationship; ET, effect type; ES, effect size

Additional (eco-)toxicological models have been developed in the last decade that extend the underlying concepts of CA: Generalized concentration addition (GCA) that extends the classical CA model to compounds with different concentration-effect curves (Howard \& Webster, 2009; Tanaka \& Tada, 2017) and the Stressor Addition Model (SAM) that focuses on predicting the effects for one chemical under different levels of an environmental stressor (Liess et al., 2016) (Table 1). The latter differs more fundamentally from CA (and GCA) in that instead of stressor-effect relationships, the density of stress capacities in a population forms the basis of the calculation. To date, SAM has only been described for mortality as effect. Mechanistically, SAM assumes that all types of stressors act on a universal stress capacity and that the joint effect is estimated by adding up the individual effects on the universal stress capacity (for details see Liess et al., 2016). By contrast, the CA model relies on adding up stressor intensities. SAM is the only approach that explicitly considers the sensitivity distribution (Figure 1a,b), measured as the density of stress capacities, which is unknown in most cases, but can be derived from the stressoreffect relationship for mortality as effect (Figure 1a). Note that if the density distribution of stress capacities is uniform, SAM predictions equal those of the simple addition model.

For application in multiple stressor studies, the presented null models require different types of input data (Figure $1 \mathrm{~b}$, Table 1).
The simple addition, multiplicative, and dominance models require information on $f_{A}\left(S I_{A}\right)$ and $f_{B}\left(S I_{B}\right)$, that is, the individual effects of the stressors $A$ and $B$ (or the related responses if directly used in a statistical model). Such data as well as data on the joint effect, which can be used to confront null model predictions with observations, are typically available from multiple stressor studies. By contrast, CA and SAM require stressor-effect relationships and sensitivity distributions, respectively. Such relationships and distributions are much less frequently available because (1) most multiple stressors studies employ too few treatment levels (see below for details) and (2) particularly in the case of SAM, few studies provide the raw data that is required to derive these.

\section{3 | STRESSOR-EFFECT RELATIONSHIPS: ISSUES OF STUDY DESIGN AND CROSS- STUDY VARIABILITY}

The majority of studies on multiple stressors employs a factorial design. To establish a reliable stressor-effect relationship from a factorial experiment, we suggest that a minimum of five treatment levels (measured on an interval or ratio scale) are needed (cf. Liess et al., 2016) given that typically at least two parameters are 
estimated (slope and intercept in a linear model or steepness and inflection point in the most parsimonious log-logistic model, which is sigmoidal; for details, see Ritz, Baty, Streibig, \& Gerhard, 2016). The number of stressor levels is rarely reported in meta-analyses, but a recent meta-analysis of multiple stressor effects on fish found that only 5 of 33 studies used five or more treatment levels of a factor (Matthaei \& Lange, 2016). Similarly, a reanalysis of studies on multiple stressors in marine systems reported that only 18 of 143 studies used five or more treatment levels of a factor (Griffen et al., 2016). While lack of data hampers the estimation of stressor-effect relationships and in turn excludes the selection of some null models, we argue that it also limits our capacity to understand and predict effects beyond the treatment levels and that this may partly explain the variability in reported interaction types in meta-analyses. To illustrate this point, we use data from a study on the production of salmon under different levels of water flow and rearing density in aquaculture conducted in 1982 (Banks, 1994). We define the water inflow of $2,271 \mathrm{~L} / \mathrm{min}$ and the rearing density of 60,000 fish as control condition with an associated adult production of 167. A water inflow of $1,514 \mathrm{~L} / \mathrm{min}\left(\mathrm{SI}_{\mathrm{A} 1}\right)$ and a density of $40,000\left(\mathrm{SI}_{\mathrm{B} 1}\right)$ led to individual reductions in adult production by approximately 20 and 40 individuals $\left(f_{A}(1,514 \mathrm{~L} / \mathrm{min})=0.5 f_{B}(40,000)\right)$, respectively, and the joint effect $f_{A B 1}(1,514 \mathrm{~L} / \mathrm{min}, 40,000)$ was a reduction in 58 adults compared to the control. The simple addition null model would relatively accurately predict the joint effect (predicted: $40+20=60$, observed: 58). Water inflow of $757 \mathrm{~L} / \mathrm{min}\left(\mathrm{SI}_{\mathrm{A} 2}\right)$ and a rearing density of $20,000\left(\mathrm{SI}_{\mathrm{B} 2}\right)$ individually led to an effect of 94 and 40 , respectively, and the joint effect was $f_{A B 2}(757 \mathrm{~L} / \mathrm{min}$, $20,000)=81$. Here, the simple additive prediction would be too high (predicted: $94+40=134$, observed: 81 ). Thus, the same stressors and experimental setting might be assigned different interaction types, that is, additive (first data set) and antagonistic (second data set), because of differences in treatment levels. Moreover, in the case of nonlinear effects (e.g. sigmoidal stressor-effect relationship), we cannot accurately predict the effects of untested intensities even of single stressors without knowing the stressor-effect relationships. For example, the effect of, for example, 1,000 or 2,000 L/min flow and of a density of 50,000 would be uncertain. Such uncertainty would be even higher for joint stressor effects. This is because joint stressor-effect relationships are very unlikely to follow the simple additive null model for all stressor combinations, and a high number of observations across the stressor gradients are required to allow for reliable prediction (Lange et al., 2014; Sett et al., 2014). For example, if for the fish study above the effect of one stressor would reduce fish production close to zero, the second stressor could only have minor additional effects on the fish production. Hence, in twostressor experiments, if one stressor approaches the effect limit, first antagonism with respect to a simple additive null model and later dominance is likely to occur.

Comparing stressor-effect relationships between studies would decrease the variability originating from different treatment levels. Although some stressor variables are difficult to quantify and defy the derivation of stressor-effect relationships (e.g. habitat alteration, alien species invasion), the majority of stressors could be implemented at many different levels in multiple stressor studies. Moreover, in situations where studies have been conducted under similar conditions, stressor-effect relationships may be inferred by pooling treatment-level effects among studies. Ultimately, this would enhance our ability to predict beyond the treatment levels of an individual study (i.e. to inter- and extrapolate, depending on the stressor and range of tested levels).

Regarding the application domain of stressor-effect relationships, we suggest that they should mainly focus on the level of individuals. This is because stressors act on this level and the established relationships are therefore presumably most robust (Hodgson, Essington, \& Halpern, 2017; Maltby, 1999). On the population and community level and above, stressor-effect relationships are influenced by multiple ecological processes such as density dependence, biotic interactions or dispersal dynamics (Vellend, 2010)(Hodgson et al., 2017). Consequently, these processes will interfere with the identification of the direct stressor effect, also termed environmental filtering, rendering results of such studies strongly context dependent (cf. Cadotte \& Tucker, 2017). Notwithstanding, many populations are density regulated (Brook \& Bradshaw, 2006; but see Knape \& de Valpine, 2012) and if the stressor-effect relationship accounts for this density regulation (e.g. by running experiments at the densities typical for the field situation or through modelling; Hodgson et al., 2017) and if meta-population dynamics can be incorporated or are irrelevant (e.g. population is isolated), extrapolation to the population level can be straightforward. Hence, we argue that while multiple stressor studies on the population and community level and above provide valuable information unless stressors are the only drivers of population regulation and community assembly, they require different theoretical frameworks for prediction and development of additional null models. Such a framework would, for example, link individual-level effects with process-based models describing population or community dynamics. Rising to this challenge, the recent development of a new model, the so-called compositional null model by Thompson et al. (2018) bases its predictions on the effects of stressors on individual species, calculated using the simple additive null model that are subsequently aggregated to the community level. This compositional null model could also be integrated with the other (individual level) null models presented for predicting the effects on individual species. Finally, statistical approaches can aid in deriving stressor-effect relationships on all levels of biological organization, supporting null model development and evaluation (Wagenhoff, Liess et al., 2017).

\section{ADEQUACY AND SELECTION GUIDELINES FOR NULL MODELS}

Studies on multiple stressors typically reject the selected null model in the case of a statistically significant interaction term. While discovery of a statistically significant interaction may explain a given phenomenon, such detection does not in itself advance our 
predictive capacity. Conversely, our predictive capacity is higher if the null model better explains the data than models including interaction terms (i.e. interaction terms not significant and model without interactions have a lower information criterion such as the AIC). The null model would ideally be selected before data analysis based on mechanistic understanding such as knowledge on the stressor mode of action (SMOA) as outlined below. Furthermore, whether an interaction that is statistically significant depends partly on the sample size of the study, which means that of two interactions with the same effect size, only the one related to the study with a higher sample size may be significant. Therefore, evaluation of the null model fit for the study data should take the size of the deviation from null model predictions into account, rather than rejecting a null model and accepting a model including interaction terms based on statistical significance or information criterion alone. Meta-analyses on multiple stressors have so far largely ignored the effect sizes of statistically significant interactions, that is, of deviations from the null model. By contrast, in the context of chemical risk assessment, the mixture models outlined above (CA and EA) were regarded as fitting as long as they deviated less than a factor of 2 from the observation (Belden, Gilliom, \& Lydy, 2007) in terms of the stressor concentration in experiments, for example, the concentration that is predicted to harm $50 \%$ of the individuals is compared to the observed concentration harming $50 \%$. Similar deviation ranges could arguably be adopted in multiple stressor studies.

The vastly different ecosystem types, organism groups and stressor modes of actions complicate defining universal rules for null model selection for prediction of the joint effects on individuals (or populations, but see previous section on the application domain of stressor-effect relationships for caveats). However, we suggest general guidelines (Figure 3) that are informed by elements of the mechanistic understanding of stressor effects such as SMOA (see glossary), correlation of sensitivities (see Figure 2), effect type and the stressor-effect relationship. These elements of mechanistic understanding are necessary for application of the guidelines and should, wherever possible, be justified from previous research. Moreover, the effect sizes of the stressors contribute to the selection of a null model for predicting the joint effect.

The first step in the application of the guidelines is to identify the effect type and the stressor intensities, for which the prediction is intended and for which effect sizes for the individual stressors (i.e. $f_{A}\left(S_{A}\right)$ and $f_{B}\left(S_{B}\right)$, Table 1) are available. Subsequently, information, if available, on the SMOA, correlation of sensitivities and stressoreffect relationships should be compiled. In the absence of knowledge on the stressor-effect relationship or the sensitivity distribution, the concentration addition and stressor addition null models cannot be applied. If the stressor-effect relationship is available and the stressors are either known or can be assumed to act similarly on organisms (i.e. same SMOA), concentration addition should be applied as the null model. In instances where neither concentration addition nor stressor addition null models can (information lacking) or should (conditions regarding SMOA or effect type not met) be applied, the next step is to check whether to apply the dominance null model
(Figure 3). The latter should be applied if the stressors are either known or can be assumed to be highly positively correlated (Figure 2) or if the effect size of any stressor is close to the effect limit (Figure 3). For example, if one of the stressors reduced the growth of a plant close to zero, the dominance model should be applied. The remaining steps provide guidance to select between the simple addition and multiplicative null model. The multiplicative model should be applied when the effect is mortality or when summing the effect sizes of the individual stressors exceeds or can be assumed to exceed the effect limit (Figure 3, cf. Figure 2 for calculation). There are likely to be exceptions to these guidelines that provide an opportunity for further testing and refinement, but in the absence of other frameworks, may inform null model selection for prediction in multiple stressor research and management. In situations where SMOA and stressor correlations are unknown and no reasonable assumptions can be made, all null models that match the remaining decision criteria (e.g. effect size) can be calculated to obtain a range of predictions instead of ignoring the uncertainty.

Finally, the relevance of selecting the correct null model will depend on the effect sizes of the stressors involved. If the effect size of one of the stressors is close to the effect limit or its effect size is approximately a factor of 10 (i.e. effect size $(E S)_{1}=10 E_{2}$ ) or more higher than that of the other stressor, the selection of the null model is likely less relevant as the null model predictions will be relatively congruent (e.g. a factor 10 difference yields: dominance $=\mathrm{ES}_{1}<$ multiplicative $<$ simple $\quad$ addition $=\mathrm{ES}_{1}+\mathrm{ES}_{2}=1.1$ $E S_{1}$ ), though exceptions may exist for SAM and CA.

\section{5 | CONCLUSIONS}

Understanding and predicting of the effects of multiple stressors presents one of the most pressing challenges in conservation and applied ecology. Rising to this challenge is a growing body of empirical research seeking to quantify multiple stressor effects, and notably, to identify instances where stressors interact yielding complex synergistic or antagonistic outcomes. While a sufficient number of studies have now permitted formal meta-analyses into the prevalence of simple (e.g. additive) and complex joint effects, due to the lack of conceptual consensus in methodical and operational definitions, studies are rarely comparable or explanatory. At the centre of this issue is the apparent absence of a mechanistic basis for null model selection and a preoccupation with detecting statistically significant interactions to the detriment of improved capacity to predict and explain (but see Wagenhoff, Liess et al., 2017; Wagenhoff, Clapcott, Lau, Lewis, \& Young, 2017). With the ultimate goal of maximizing our ability to predict and explain effects, multiple stressor research must move towards mechanistically justified selection and fitting of null models - to which we contributed here - typically on the level of individuals and populations. Managing ecosystems in the face of global change requires understanding and predicting how multiple stressor effects scale from individuals to ecosystems, justified selection of the appropriate null model is a first step to achieving this. 


\section{ACKNOWLEDGEMENTS}

RBS acknowledges funding by the DFG (SCHA 1720/17-1) and the EU (Project SERIOR). Mira Kattwinkel and two anonymous reviewers provided valuable comments that improved the manuscript.

\section{ORCID}

Ralf B. Schäfer (iD http://orcid.org/0000-0003-3510-1701

\section{REFERENCES}

Backhaus, T., \& Faust, M. (2012). Predictive environmental risk assessment of chemical mixtures: A conceptual framework. Environmental Science \& Technology, 46, 2564-2573. https://doi.org/10.1021/ es2034125

Banks, J. L. (1994). Raceway density and water flow as factors affecting spring chinook salmon (Oncorhynchus tshawytscha) during rearing and after release. Aquaculture, 119, 201-217. https://doi.org/10. 1016/0044-8486(94)90176-7

Belden, J. B., Gilliom, R. J., \& Lydy, M. J. (2007). How well can we predict the toxicity of pesticide mixtures to aquatic life? Integrated Environmental Assessment and Management, 3, 364-372. https://doi.org/10. 1002/(ISSN)1551-3793

Bliss, C. I. (1939). The toxicity of poisons applied jointly. Annals of Applied Biology, 26, 585-615. https://doi.org/10.1111/j.1744-7348.1939.tb 06990.x

Brook, B. W., \& Bradshaw, C. J. A. (2006). Strength of evidence for density dependence in abundance time series of 1198 species. Ecology, 87, 1445-1451. https://doi.org/10.1890/0012-9658(2006)87[1445: SOEFDD]2.0.CO;2

Brudvig, L. A. (2017). Toward prediction in the restoration of biodiversity. Journal of Applied Ecology, 54, 1013-1017. https://doi.org/10.1111/ 1365-2664.12940

Cadotte, M. W., \& Tucker, C. M. (2017). Should environmental filtering be abandoned? Trends in Ecology \& Evolution, 32, 429-437. https://d oi.org/10.1016/j.tree.2017.03.004

Cedergreen, N. (2014). Quantifying synergy: A systematic review of mixture toxicity studies within environmental toxicology. PLOS ONE, 9, $1-12$.

Côté, I. M., Darling, E. S., \& Brown, C. J. (2016). Interactions among ecosystem stressors and their importance in conservation. Proceedings of the Royal Society B: Biological Sciences, 283, 20152592.

Crain, C. M., Kroeker, K., \& Halpern, B. S. (2008). Interactive and cumulative effects of multiple human stressors in marine systems. Ecology Letters, 11, 1304-1315. https://doi.org/10.1111/j.1461-0248.2008. 01253.x

Dunne, R. P. (2010). Synergy or antagonism - interactions between stressors on coral reefs. Coral Reefs, 29, 145-152. https://doi.org/10. 1007/s00338-009-0569-6

Escher, B. I. (2013). Modes of Action of Chemical Pollutants. In J.-F. Férard, \& C. Blaise (Eds.), Encyclopedia of Aquatic Ecotoxicology (pp. 737-750). Dordrecht, the Netherlands: Springer. https://doi.org/10. 1007/978-94-007-5704-2

Folt, C. L., Chen, C. Y., Moore, M. V., \& Burnaford, J. (1999). Synergism and antagonism among multiple stressors. Limnology and Oceanography, 44, 864-877. https://doi.org/10.4319/lo.1999.44.3_part_2.0864

Griffen, B. D., Belgrad, B. A., Cannizzo, Z. J., Knotts, E. R., \& Hancock, E. R. (2016). Rethinking our approach to multiple stressor studies in marine environments. Marine Ecology Progress Series, 543, 273-281. https://doi.org/10.3354/meps11595
Hale, R., Piggott, J. J., \& Swearer, S. E. (2017). Describing and understanding behavioral responses to multiple stressors and multiple stimuli. Ecology and Evolution, 7, 38-47. https://doi.org/10.1002/ece3. 2609

Hodgson, E. E., Essington, T. E., \& Halpern, B. S. (2017). Density dependence governs when population responses to multiple stressors are magnified or mitigated. Ecology, 98, 2673-2683. https://doi.org/10. 1002/ecy.1961

Holmstrup, M., Bindesbol, A. M., Oostingh, G. J., Duschl, A., Scheil, V., Köhler, H. R., ... Spurgeon, D. J. (2010). Interactions between effects of environmental chemicals and natural stressors: A review. Science of the Total Environment, 408, 3746-3762. https://doi.org/10.1016/j. scitotenv.2009.10.067

Howard, G. J., \& Webster, T. F. (2009). Generalized concentration addition: a method for examining mixtures containing partial agonists. Journal of Theoretical Biology, 259, 469-477. https://doi.org/10.1016/ j.jtbi.2009.03.030

Jackson, M. C. (2015). Interactions among multiple invasive animals. Ecology, 96, 2035-2041. https://doi.org/10.1890/15-0171.1

Jackson, M. C., Loewen, C. J. G., Vinebrooke, R. D., \& Chimimba, C. T. (2016). Net effects of multiple stressors in freshwater ecosystems: A meta-analysis. Global Change Biology, 22, 180-189. https://doi.org/ 10.1111/gcb.13028

Kendler, K. S., \& Gardner, C. O. (2010). Interpretation of interactions: Guide for the perplexed. The British Journal of Psychiatry, 197, 170 171. https://doi.org/10.1192/bjp.bp.110.081331

Knape, J., \& de Valpine, P. (2012). Are patterns of density dependence in the Global Population Dynamics Database driven by uncertainty about population abundance? Ecology Letters, 15, 17-23. https://doi. org/10.1111/j.1461-0248.2011.01702.x

Kroeker, K. J., Kordas, R. L., \& Harley, C. D. G. (2017). Embracing interactions in ocean acidification research: Confronting multiple stressor scenarios and context dependence. Biology Letters, 13, 20160802. https://doi.org/10.1098/rsbl.2016.0802

Lange, K., Townsend, C. R., \& Matthaei, C. D. (2014). Can biological traits of stream invertebrates help disentangle the effects of multiple stressors in an agricultural catchment? Freshwater Biology, 59, 2431-2446. https://doi.org/10.1111/fwb.12437

Lewis, S. L., \& Maslin, M. A. (2015). Defining the Anthropocene. Nature, 519, 171-180. https://doi.org/10.1038/nature14258

Liess, M., Foit, K., Knillmann, S., Schäfer, R. B., \& Liess, H.-D. (2016). Predicting the synergy of multiple stress effects. Scientific Reports, 6, 32965. https://doi.org/10.1038/srep32965

Loewe, S., \& Muischnek, H. (1926). Über Kombinationswirkungen. Naunyn-Schmiedebergs Archiv für experimentelle Pathologie und Pharmakologie, 114, 313-326. https://doi.org/10.1007/BF01952257

Maltby, L. (1999). Studying stress: The importance of organism-level responses. Ecological Applications, 9, 431-440. https://doi.org/10. 1890/1051-0761(1999)009[0431:SSTIOO]2.0.CO;2

Matthaei, C. D., \& Lange, K. (2016). Multiple-stressor effects on freshwater fish: A review and meta-analysis. In G. P. Closs, M. Krkosek, \& J. D. Olden (Eds.), Conservation of freshwater fishes (pp. 178-214). Cambridge, UK: Cambridge University Press. https://doi.org/10.1017/ CBO9781139627085

Piggott, J. J., Salis, R. K., Lear, G., Townsend, C. R., \& Matthaei, C. D. (2015). Climate warming and agricultural stressors interact to determine stream periphyton community composition. Global Change Biology, 21, 206-222. https://doi.org/10.1111/gcb.12661

Piggott, J. J., Townsend, C. R., \& Matthaei, C. D. (2015). Reconceptualizing synergism and antagonism among multiple stressors. Ecology and Evolution, 5, 1538-1547. https://doi.org/10.1002/ece3.1465

Quinn, G., \& Keough, M. (2002). Experimental design and data analysis for biologists. Cambridge, UK: Cambridge University Press. https://doi. org/10.1017/CBO9780511806384 
Ritz, C., Baty, F., Streibig, J. C., \& Gerhard, D. (2016). Dose-response analysis using R. PLOS ONE, 10, 1-13.

Schäfer, R. B., Kühn, B., Malaj, E., König, A., \& Gergs, R. (2016). Contribution of organic toxicants to multiple stress in river ecosystems. Freshwater Biology, 61, 2116-2128. https://doi.org/10.1111/fwb.12811

Sett, S., Bach, L. T., Schulz, K. G., Koch-Klavsen, S., Lebrato, M., \& Riebesell, U. (2014). Temperature modulates coccolithophorid sensitivity of growth, photosynthesis and calcification to increasing seawater pCO2. PLOS ONE, 9, 1-9.

Tanaka, Y., \& Tada, M. (2017). Generalized concentration addition approach for predicting mixture toxicity. Environmental Toxicology and Chemistry, 36, 265-275. https://doi.org/10.1002/etc.3503

Thompson, P. L., MacLennan, M. M., \& Vinebrooke, R. D. (2018). An improved null model for assessing the net effects of multiple stressors on communities. Global Change Biology, 24, 517-525. https://d oi.org/10.1111/gcb.13852

Vellend, M. (2010). Conceptual synthesis in community ecology. The Quarterly review of biology, 85, 183-206. https://doi.org/10.1086/ 652373

Vinebrooke, R. D., Cottingham, K. L., Norberg, J., Scheffer, M., Dodson, S. I., Maberly, S. C., \& Sommer, U. (2004). Impacts of multiple stressors on biodiversity and ecosystem functioning: The role of species co- tolerance. Oikos, 104, 451-457. https://doi.org/10.1111/j.00301299.2004.13255.x

Wagenhoff, A., Clapcott, J. E., Lau, K. E. M., Lewis, G. D., \& Young, R. G. (2017). Identifying congruence in stream assemblage thresholds in response to nutrient and sediment gradients for limit setting. Ecological Applications, 27, 469-484. https://doi.org/10.1002/eap.1457

Wagenhoff, A., Liess, A., Pastor, A., Clapcott, J. E., Goodwin, E. O., \& Young, R. G. (2017). Thresholds in ecosystem structural and functional responses to agricultural stressors can inform limit setting in streams. Freshwater Science, 36, 178-194. https://doi.org/10.1086/ 690233

How to cite this article: Schäfer RB, Piggott JJ. Advancing understanding and prediction in multiple stressor research through a mechanistic basis for null models. Glob Change Biol. 2018;00:1-10. https://doi.org/10.1111/gcb.14073 